\title{
SUPPLEMENTA PRAEFATIONIS
}

I. De codicis $\mathrm{C}$ apographis.

Apographa mibi innotuerunt haec:

P Paris. graec. 1894 chart. saec. XVI (Omont II 160)

Q Paris. graec. 1895 chart. saec. XV/XVI (Omont l. c.)

A Paris. graec. 1896 chart. saec. XV; ef. p. VI

R Paris. graec. 1897 chart. saec. XVI (Omont l. e.)

S Paris. graec. 1912 chart. saec. XVI (Omont II 162)

T Paris. graec. 1926 chart. saec. XVI (Omont II 165)

U Paris. graec. 2354 chart. saec. XVI (Omont II 245)

M Montepessulanus scholae medicorum n. 64 (Omont III 374)

E Estensis Mutin. III B 14 chart. saec. XV

L Laurentianus 85,27 membr. saec. XV

I Palat. gr. 63 chart. saec. XV

K Vatic. gr. 1473 chart. saec. XVI

$\mathrm{N} \quad$ Neapolit. III $\& 11$ saec. XV

Escorial. T II 16 ehart. saec. XVI

Matritens. bibl. nat. 027 chart. saec. XVI

B Bodleian. misc. 194 chart. anni 1541

Mosquensis de quo Matthaei accurata codd. graec. bibl. Mosqu. notitia p. $9 \mathrm{sq}$.

H Hamburgensis de quo ef. p. VI.

Rettulerunt de Parisinis C. Hoelk, de Montepessulano M. Bonnet, de Estensi Laurentiano Neapolitano A. Gercke, de Palatino et Vaticano E. Pfuhl et L. Deubner, de Bodleiano H. Rabe. varias lectiones enoto ad initium pertinentes omissis eis, quae ad orthographiam spectant.

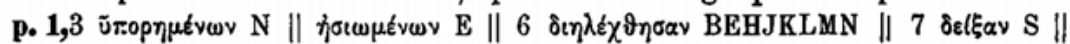

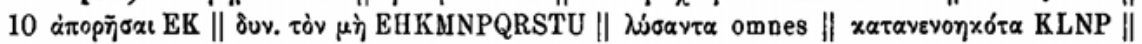

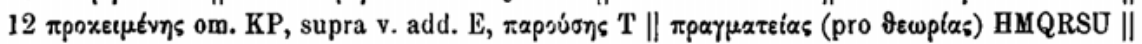

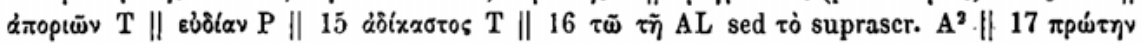

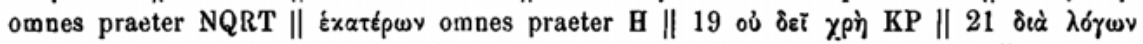

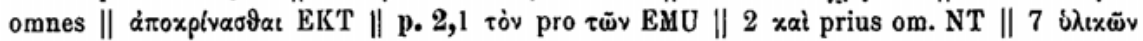

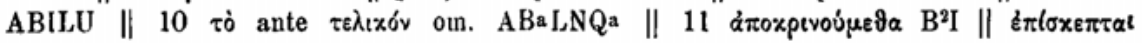

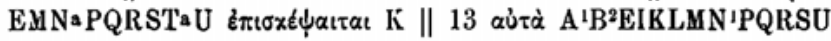


Haec satis esse opinor ad naturam horum codicum cognoscendam; de quibus si quis plura discere cupiet, libenter ei schedas meas transmittam.

\section{Varia lectio Aristotelea.}

Ipsa Aristotelis verba, quae lemmatis continentur, cum in collatione mihi tradita respecta nou essent, postea demum Syriani textu iam impresso codicem inspexi et lemmata contuli. liceat igitur hoe loco lectiones diserepantes enotare, quae ad libros B M N pertinent; repeto autem eas quoque quae iam in adnotatione critica indicatae sunt, ut omnes sub uno conspectu prostent. utor Christii editione anno 1886 emissa; sed ad librum B etiam Bekkeri apparatum adhibui, qui ad hune librum ipsum codicem C contulit, eam scilicet partem, in qua Metaphysica extant (fol. 226-409), notaque $I^{b}$ distinxit. et quantum iudicare licet - Bekkerus enim saepius notas confudisse videtur: ef. Bonitz I p. VIII lemmata Syrianea codicis $\mathrm{C}$ ex eiusdem libri parte priore i. e. ex $\mathrm{I}^{\mathrm{b}}$ aut ex eodem archetypo ex quo illa descripta esse videntur. ${ }^{1}$ )

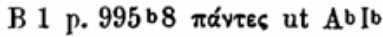
$23 \pi \tilde{\alpha} \sigma \alpha \nu$ ut $\mathrm{AbIb}: \alpha \pi \kappa \sigma \alpha \nu \mathrm{E}$

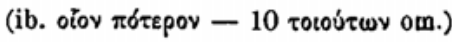

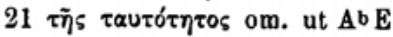

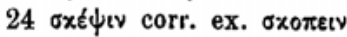

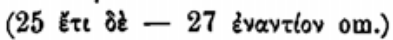
$28 \tau \dot{\alpha}$ ante $\gamma^{\hat{\varepsilon}} v \eta$ om. ut $\mathrm{Ib}$
34 tồóv Ėơt ut $\mathrm{Ib}$ : żờì tồtov $\mathrm{A}^{\mathrm{b}} \mathrm{E}$

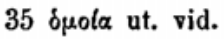

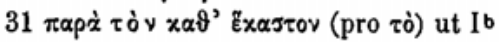

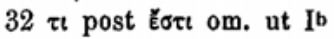

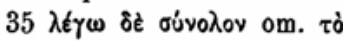

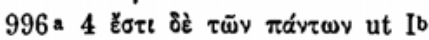
6 Ėerov ut $\mathrm{E}$ supra lin, et Ib

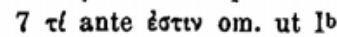
8 y pol om. ut Ib

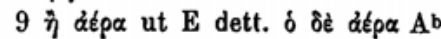

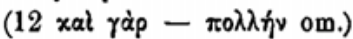

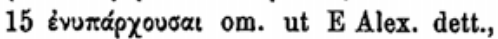 habet $\mathrm{A}^{\mathrm{b}}$

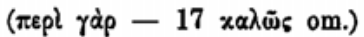

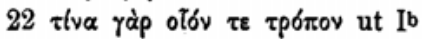

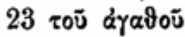

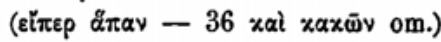

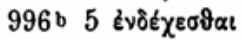

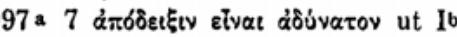

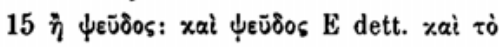 $\psi \varepsilon \overline{0} \delta 0$ s Ab

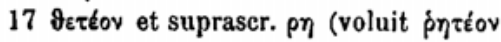 ut Ib)
23 ชò $8 \tau \iota$ ut $\mathrm{E}$ plerique: $8 \mathrm{Ab}$ Alex.

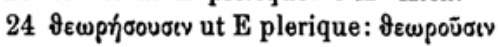 $\mathrm{A}^{\mathrm{b}}$

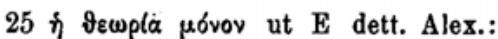

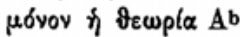
$997 \mathrm{~b} 10 \mathrm{om}$. etvat ut $\mathrm{E}$ dett.: habet $\mathrm{Ab}$
$11 \pi 0{ }^{2} 0 \tilde{\sigma} \sigma$ (sic Ib) et suprascr. Ėroiouv
$17 \tau \varepsilon$ om. ut I

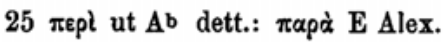
27 at $\mathrm{E}$ dett.: ๘ँv $\mathrm{Ab}$
$29 \tau \ddot{\eta} \leqslant$ pro $\alpha \dot{u} \tau \tilde{\eta}_{s}$ ut $\mathrm{I}^{\mathrm{b}}$
$31 \pi \alpha \rho \dot{\alpha}$ et suprascr. epl ( $\pi \varepsilon p i \mathrm{~Gb}$ )

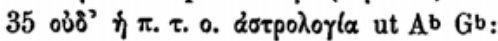

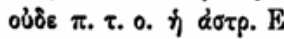

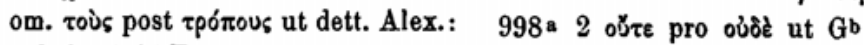 habent $\mathrm{A}$ bE

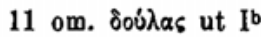

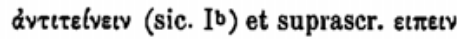

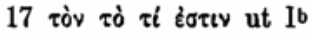
5 ai Ėtxes ut E: $\alpha$ i om. Ab
$\omega$

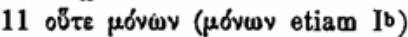

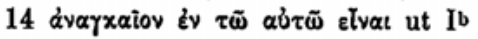

1) Omnino eiciendus est Bekkeri Gb; ęst ẹnim Syriani cod. Paris. 1896 (cf. p. VII), qui totus fluxit ex C (Ib). 


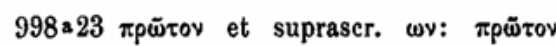
$\mathrm{AbE}^{1} \pi \rho \dot{\omega} \tau \omega$ v $\mathrm{E}^{2}$ Alex.

24 om. $\tau \alpha \tilde{v} \tau^{\prime}$ ut Ib

$30 \mu \varepsilon \tau \dot{\alpha}$ ut $\mathrm{E}$ dett.: $\mu \varepsilon \tau \alpha \xi j \mathrm{j} \mathrm{A}^{\mathrm{b}}$

998b 1 \& $9 \lambda_{1}$ ot ut Ib

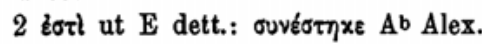

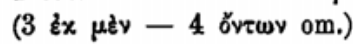

4 el ut Ab Ascl.: $\eta^{\circ} \mathrm{E}$

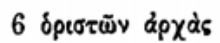
$x \alpha$ ut $\mathrm{A}^{\mathrm{b}}$ dett.: $x \not \partial \vee \mathrm{E}$

$9 \tau \tilde{\omega}$ ante $\lambda \varepsilon \gamma \delta$ б $\tau \omega \nu$ ex corr.

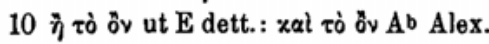

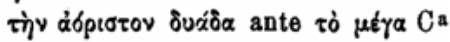
del. $\mathrm{Cb}$

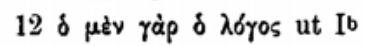

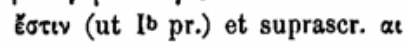

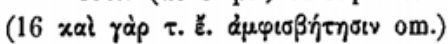

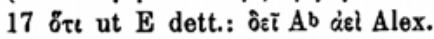

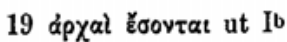

20 ồ ex corr.

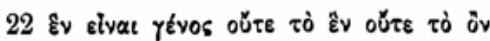
ut $\mathbf{E}$ dett.

23 in $\mu \dot{z} v$ yà

24 ydp pro ồ ut Ib

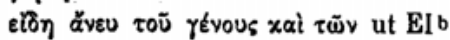

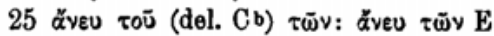

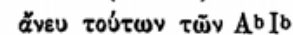

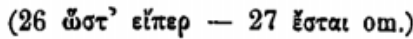

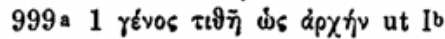

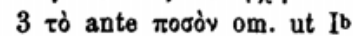

4 ótatpeizat ut Ib

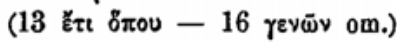

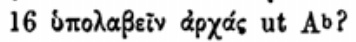

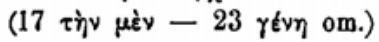

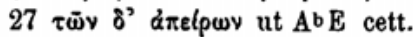

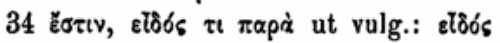
$\tau$ om. $\mathrm{Ab} \mathrm{E}$

999b 1 oùtv et ita plerumque

$3 \lambda$ eryoc ut Ib

ह้นเ ut $\mathrm{E}$ dett.: ह̌ँ

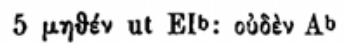

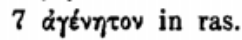

8 om. $\tau \varepsilon$

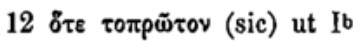

13 àyéviros ut $\mathrm{AbE}$ cett.

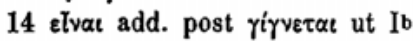

$21 \pi \alpha \dot{v} \tau \alpha$ ut Ib

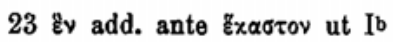

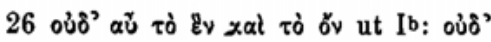

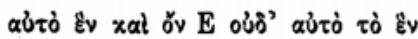
xal $\tau \dot{0}$ b̆ $\mathrm{A}^{\mathrm{b}}$
$27-1000$ a 4 cf. p. 40,23

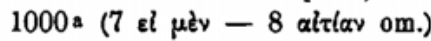

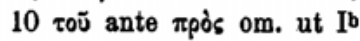

12 גußpwoias

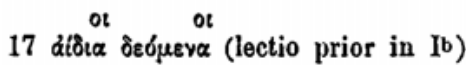

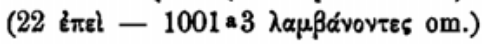

1001 a 4 xat post $\delta \hat{z}$ om. ut Ib

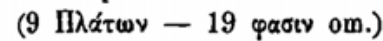

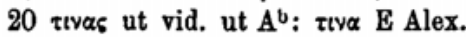

24 ö $\pi$ pro हैंt ut Ib

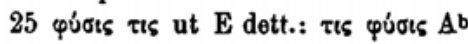

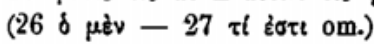

27 aùroèv xal aũroòv ut Ib

1001 a $28 \times \alpha 96$ तou om. ut Ib

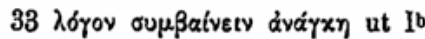

1001 b 4 ràp xaì ut $\mathrm{E}$ dett.: xaì $\mathrm{om}$. Ab

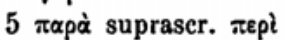

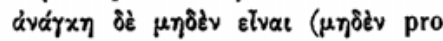
$\mu \eta े$ \&े etiam $\mathrm{E}$ dett.)

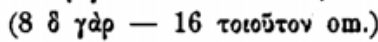

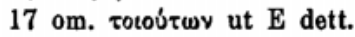

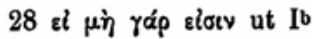

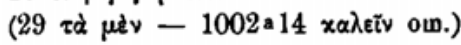

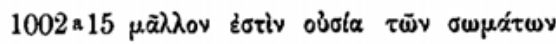
$\tau \dot{\alpha} \mu \gamma \not \eta \eta$ ut dett.

19 om. Toü ut $\mathrm{I}^{\mathrm{b}}$

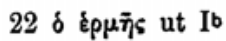

$24 \varepsilon$ l' pro in ut Ib

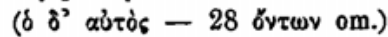

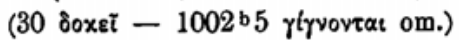

1002 b 6 om. $\tau \bar{\varphi}$ ut Ib

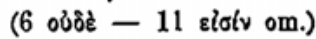

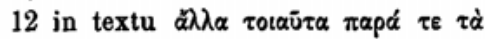

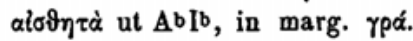

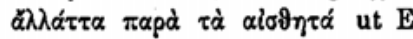

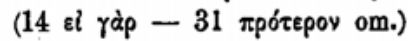

(1003 a $1-2$ aitias om.)

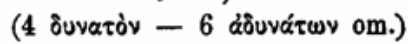

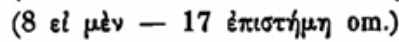

Libri $\Gamma$ solum initium extat, in quo

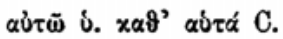

Liber $M$ incipit a p. 1076 $\$ 38$ (cf. tamen ad p. 84,6).

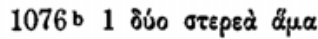

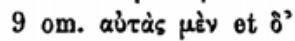

11 cf. p. 87,1

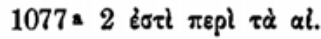

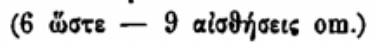

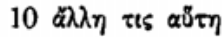




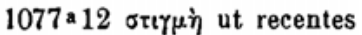

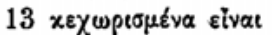

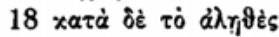

$19 \tau \bar{\eta} \gamma \varepsilon v \in \bar{t} \sigma \varepsilon t$ $\tau \tilde{\eta} \delta^{2}$ oùrka

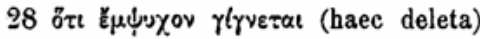

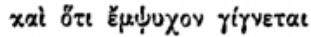

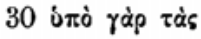

32 ठ̇, suprascr. $\delta$ iे

35 ย่ $\alpha \tau \tau \gamma \mu \bar{\omega} \nu$

36 oิuváu. vvov

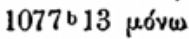

$18 \pi$ rap̀̀ (sic Alex.) del. et suprascr. $\pi \varepsilon p l\left(\right.$ sic $\mathrm{A}^{\mathrm{b}} \mathrm{E}$ )

32 oĩov $\tau \dot{\alpha}$ xtvoú $\mu \varepsilon v \alpha$ (ev $\alpha$ ex corr.)

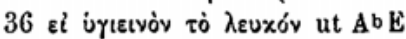
நं $\delta^{\prime}$ ěotev írectóv ut $\mathrm{A}^{\mathrm{b}}, \mathrm{E}$ in textu

1078 a 1 \&

11 om. $\mu \tilde{\alpha} \lambda \lambda$, v

$13 \delta \mu \alpha \lambda \eta_{s}$ ut E Alex.

$16 \mathrm{om}$. ố

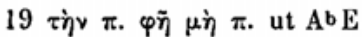

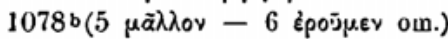

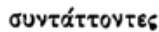

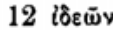

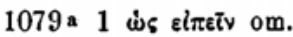

$2 \pi \alpha \rho^{\prime}$ " हx $x \sigma \tau \tau \nu \nu$

3 habet $\tau \varepsilon$

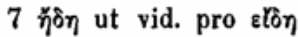

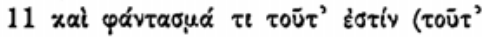
etiam E)

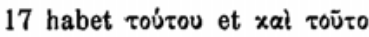

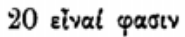

22 Ě $\sigma \tau \alpha$ ut $\mathrm{E}$, sed addit $+\delta_{\sigma \alpha \alpha} \mu \grave{\eta}$

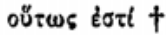
xal al ut $\mathrm{E}$

23 Eัбovtal ut $\mathrm{Ab} \mathrm{E}$

$28 \lambda$ kyoviat ut $\mathrm{AbE}$

35 ow. Elval ut $\mathrm{Ab} \mathrm{E}$

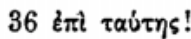

1079b $1 \times a \lambda$ oin

4 om. тò̀

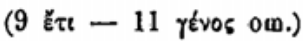

14 om. Toĩs

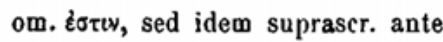

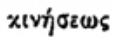

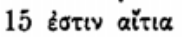
oủồ

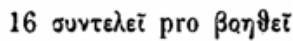

19 oü $\tau \omega s$ pro iows

$25 \delta \hat{\varepsilon} \gamma \varepsilon$ 1079b28 om. 8็potov ut AbE

29 тоர̃ ซwxpátous

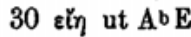

32 xat o aùr

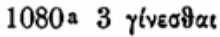

8 o $t^{\prime}$ olas

$26 \mathrm{om}$. al ut $\mathrm{E}$

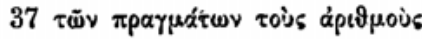

1080 b 4 मे $\pi \alpha^{\prime} v \tau \alpha s$ om. ut codd. dett.

9 eivat om. ut Ab

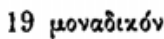

24 prius $\pi e p l$ om.

$25 \tau \tilde{\omega} \nu$ pro $\tau \dot{\alpha}$

32 है $\chi 0 v \tau \alpha$ ut $\mathrm{A}^{\mathrm{b}} \mathrm{E}$ pr.

1081 a 7 habet roi.

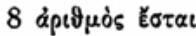

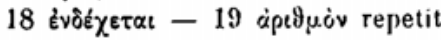

26 om. $\dot{\eta}$ et prius $\tau \dot{\eta} s$

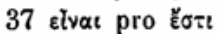

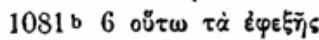

12 ăv $\tau \varepsilon$

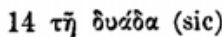

28 om. ä $2 \lambda \alpha \mathrm{t}$

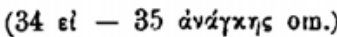

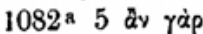

8 हैंovtat ut Alex.

9 om. al ut $\mathrm{E}$

ठ̀̀ pro ò̀

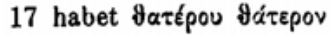

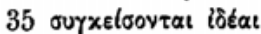

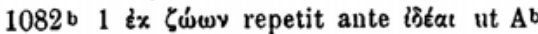

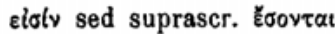

$5 \tau \varepsilon$ xal $\eta े$ toov

10 om. $\lambda \dot{z} \gamma \in t v$

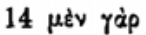

24 om. aùtò

$36 \tau \dot{\alpha} \varsigma \mu \varepsilon p(\delta \hat{\alpha} \alpha$

1083 a $4 d \lambda \lambda^{\prime}$ iे

om. tò ut $\mathrm{E}$

7 habet $\mu . \varepsilon$ ļous $\ddot{\eta}$

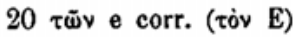

25 habet $\tau \dot{0}$

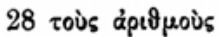

35 Qे $\delta^{\prime} \alpha \tilde{v}$

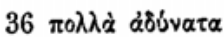

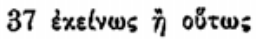

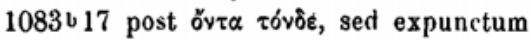

27 úrápxet ut $\mathrm{AbE}$

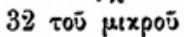

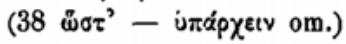

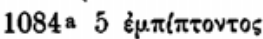




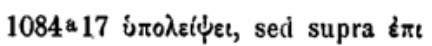

19 om. ảंtoiss

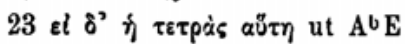

28 ow. äp $\alpha$

29 habet $\delta$

$30 \mathrm{om}$. $\tau$ ò हैv ut $\mathrm{A}^{\mathrm{b} E}$

$37 \pi \varepsilon \mu \pi \dot{s} s$ sed supra $\pi \varepsilon v \tau \alpha$ s

1084b 9 ou. $\eta$

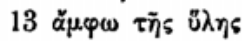

$15 \mu \dot{\varepsilon} \rho \circ \varsigma^{2}$

$19 \mu$ épos pro elరิos

20 om. xal ut $\mathbf{E}$

28 xal iो povds

33 ò̀ pro $\delta \varepsilon i$

$37 \mathrm{om}$. $\dot{\eta}$

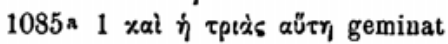

$2 \grave{\eta} \ddot{\alpha} \mu \varphi \omega$

4 бँw sed supra scr. ov

7 iotépwv

17 om. xaì ante ai

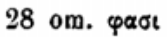

$\delta เ \alpha \lambda \bar{u} \sigma \alpha \iota$

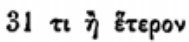

1085 b 1 om. Tõ̃

$3 \tau \alpha \bar{j} \tau \alpha$ ut $\mathrm{Ab}$, suprascr. $\tau \alpha u \tau \dot{\alpha}$ ut $\mathrm{E}$

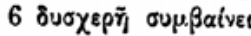

$11 \dot{\alpha} \lambda \lambda^{3} \dot{\alpha} \pi$. al aủ ${ }^{2}$

$14 \delta \dot{\partial} \dot{\eta} \varepsilon^{\prime} x$ ut $A^{b}$, suprascr. $\delta \dot{\eta} \varepsilon^{2} x$ ut $\mathrm{E}$

17 om. $\gamma^{\prime}$

$18 \mathrm{om} . \tau \varepsilon$

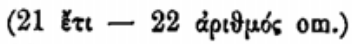

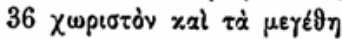

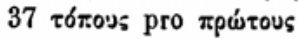

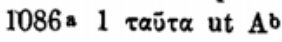

6 els pro $\varepsilon l$ ut $\mathrm{E}$, sed nescio quid supraser.

9 lóéas ut $\mathrm{E}$

$11 \tau \dot{\alpha} \tau \varepsilon \varepsilon i \hat{i} \eta$ ut dett. post $\varepsilon i \bar{\partial} \eta$ हival, sed del.

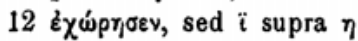

$19 \partial_{\nu}$ ante $\varepsilon x$, non ante हैं

$20 \pi \varepsilon \pi \varepsilon t \gamma \mu \dot{k}$ Vos ut $\mathrm{AbE}$

1086b 2 om. $\tau \varepsilon$

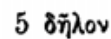

$19 \tau$ เขัं ut $\mathrm{E}$

1087 a $1 \mathrm{om}$. ai ut $\mathrm{E}$

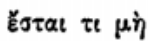

6 habet xal iốx

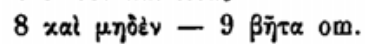

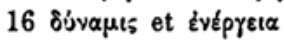

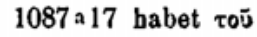

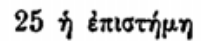

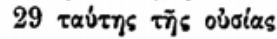

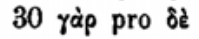

31 xaì sqq. om., cf. p. 165,24

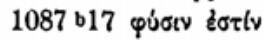

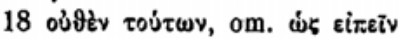

22 xal tò ut Ald.

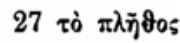

$28 \mu \hat{\varepsilon} \lambda \varepsilon t$

30 om. $\tau \tilde{\omega}$ ut $\mathrm{F}$

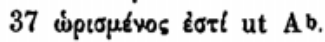

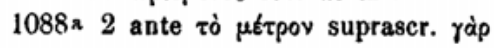

$12 \tau \bar{\omega} \alpha \dot{\omega} \tau \tilde{\omega}$

$13 x \alpha \tau^{\prime} \dot{\alpha} \mathrm{pt \vartheta \mu} \dot{\partial} \mathrm{v}$

$23 \mathrm{om} . \tau \iota s \dot{\eta े ~ o u ̀ \sigma i \alpha ~}$

25 ஜे pro el ut Alex.

$29 \mathrm{om}$. ஜ̆ ante rрós $\tau$

$30 \mu$ vou ut $\mathrm{E}$

$33 \dot{\alpha} \pi \lambda \tilde{\eta}$ supraser. $\tilde{\omega} \varsigma$

35 ह̇ $\tau i$ ut $\mathrm{E}$

$1088^{\text {b }} 3$ bè pro oủv

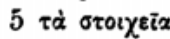

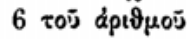

$8 \mathrm{om}$. xaì ante $\pi \lambda x \tau \dot{~}$ w̃v pro oũ.

$10 x \not ૈ v$ ยใ

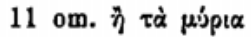
oũนos ut $\mathrm{E}$

13 ou $\mu$ óvov

18 oủồ $\gamma$ dेp

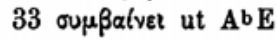

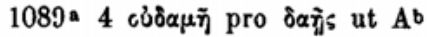

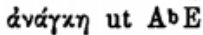

$10 \pi \alpha ́ v \tau \alpha$

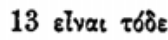

27 babet tò

33 ò pro $\delta \dot{\eta े}$

$1089 \mathrm{~b} 8 \tau \dot{\alpha}$ pro $\tau \dot{o}$

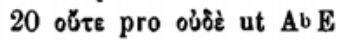

$21 \varepsilon i$ post $\check{\omega} \sigma \pi \varepsilon \rho$, sed expunctum

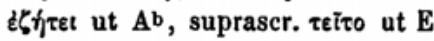

32 \&l xat

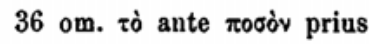

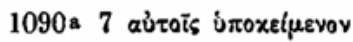

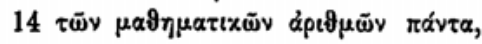

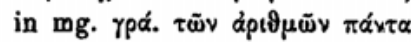

17 habet $\lambda \alpha \mu \beta \alpha$ vetv

33 oủồ ut $\mathrm{E}$

$37 \tau \iota$ pro $\tau \varepsilon$

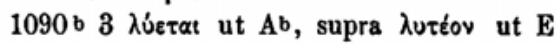


1090b 6 om. ข०ธั

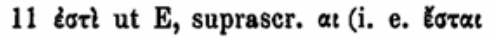
ut $\mathrm{Ab}^{\mathrm{b}}$ )

13 Eัбrat ut $A b$

14 post $\mu \dot{\varepsilon} v$ oùv, ved expunctum

25 трб́твроv

27 xal ante oủoe, sed deletum

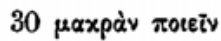

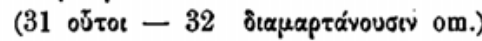

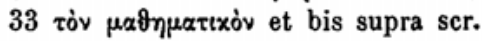
$\tilde{\omega} \nu$ (cf. $A^{b}$ )

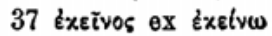
เซิะธีท

1091 a 2 Épei posterius expunctum

3 om. $\tau \varepsilon$

$4 \tau \dot{\alpha}$ हैv ut $\mathrm{A}^{\mathrm{b}}$

$12 \delta เ \pi \lambda \alpha \sigma \iota \alpha \zeta \delta \mu \varepsilon v \sigma v$, suprascr. os

14 où post iे, sed expunctum

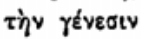

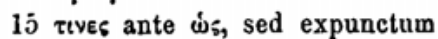

i7 habet $8 \pi \mathrm{r}$

19 pro $\delta$ ixatov est $\delta .$. . (quattuor fere fere litt. erasae)

$\tau t$ rubro in $\tau \dot{\alpha}$ corr. vid.

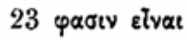

$24 \pi \rho \tilde{\omega}$ tov expunctum

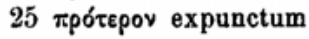

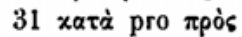

33 áóptotov

1091b 4 of pro ñ

6 xat supra $\ddot{\eta}$

$9 \pi \alpha \dot{v} \tau \tau \alpha$

$21 \gamma \varepsilon$ pro $\tau \varepsilon$ $\sigma \tau o t \chi \varepsilon(\omega v$ àptôróv

$28 \mu \delta v \omega v$

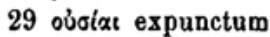

$1091 \mathrm{~b} 32$ xal $\mu \xi \gamma \alpha$ et supra $\varepsilon i \tau \varepsilon \mu \xi \gamma \alpha$

33 ws dvarxaiov ơv expunctum

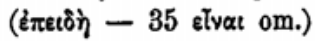

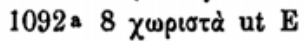

$16 \alpha \dot{u} \tau \alpha ́$ post $\tau \alpha \tilde{u} \tau \alpha$, sed expunctum

$26 \sigma \nu \lambda \lambda \alpha \beta \alpha \ell$, suprascr. $\eta^{v}\left(\right.$ ut $\mathrm{A}^{\mathrm{b}}$ )

28 हैชซนใ ỡ ut $\mathrm{Ab}$

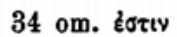

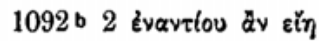

हैotเv ut $\mathrm{AbE}$

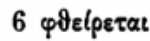

9 aǐtiov of ante öpot, sed expunctum

14 habet $\delta$, sed expunxit iे ut $\mathrm{A}^{\mathrm{b}}$

19 iो ut $A^{b}$

$22 \mu(\xi \varepsilon \omega \nu$

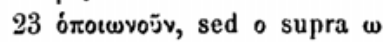

27 tò ut $\mathrm{A}^{\mathrm{b}} \mathrm{E}$

$33 \pi 0 \lambda \lambda \alpha \pi \lambda \alpha \alpha \sigma \dot{\sigma} \sigma \varepsilon \sigma \iota v$ ut. Alex.

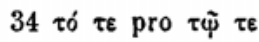

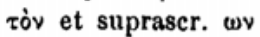

1093 a 12 om. $\tau \dot{\alpha}$

14 ह่v suprascr. m. rec.

16 om. $\pi \dot{\varepsilon} \varphi \varphi \cup x v$

20 habet xat

22 tò yà̀

$24 \tau \bar{\omega}$ ut $\mathrm{A}^{\mathrm{b}} \mathrm{E}$

28 ơ $\tau$ ut $\mathrm{AbE}^{\mathrm{b}}$

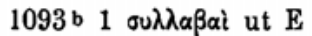

$3 \beta$ pro $\Omega$

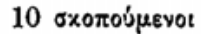

11 éxeivo pro is

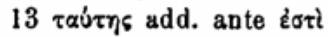

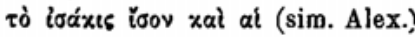

$22 \dot{\alpha} \lambda \lambda \dot{\lambda} \lambda \omega \mathrm{v}$ Ėxeivot

III. Scholia. ${ }^{1}$ )

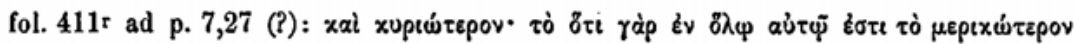
aĩtov.

fol. 424r ad p. 94,14 vide in adn.

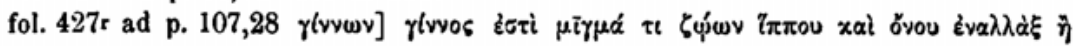

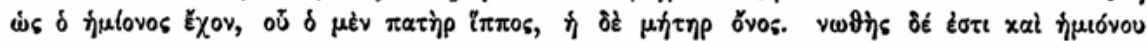

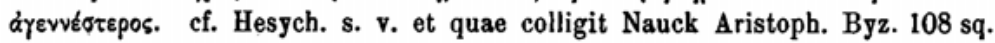
$\pi \rho \circ \beta \lambda \eta \eta_{\mu \alpha \alpha \alpha .}$

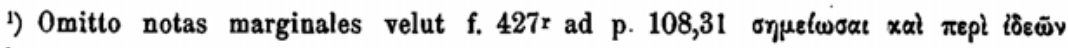




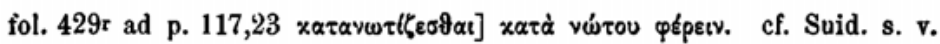

fol. 434v ad p. 142,15 sub textu editum.

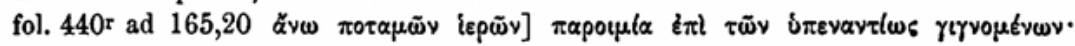

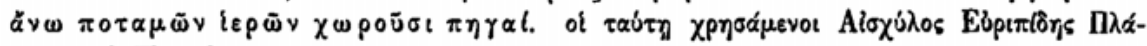
$\tau \omega v$. cf. Hesych. s. v.

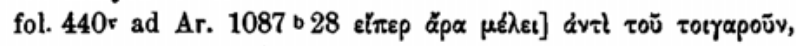

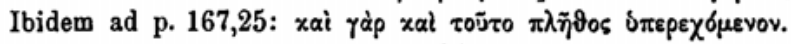

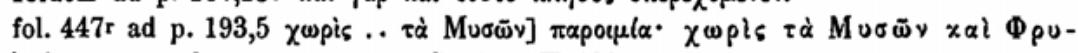
$\gamma \tilde{\omega} \nu \delta \rho / \sigma \mu \alpha \tau \alpha$. ef. corpus paroem. Gotting. II 130. 



\section{S Y R I A N I}

\section{IN METAPHYSICA}

\section{COMMENTARIA}


